\section{Avoidance conditioning of general activity in the goldfish*}

\section{PHILIP GREENWAY, University of Aberdeen, Aberdeen, Scotland AB9 2UB and \\ M. E. BITTERMAN, Univensity of Hawaii, Honolulu, Hawaii 96822}

Goldfish were trained with light as CS and brief shock as US under conditions in which specified amounts of general activity prevented shock both for experimental animals and for their yoked controls. The relation of the results to those of some recent shuttlebox experiments are considered.

Goldfish trained in a shuttlebox with light as CS and brief shock as US acquire the shuttling response to light whether or not the response prevents shock-that is, whether an avoidance or a Pavlovian procedure is used (Woodard \& Bitterman, 1971). The Pavlovian results can be explained in terms of the conditioning of general activity: light paired with shock comes to activate the animal (Horner, Longo, \& Bitterman, 1960), which then is more likely to cross the hurdle. It has been suggested also that the avoidance results can be explained in the same terms (Woodard \& Bitterman, in press). Despite a lower overall probability of shock, the level of activation by the CS may be as high in avoidance animals as in Pavlovian animals when sensory feedback (FS) from the avoidance response is readily discriminable; CS-alone is consistently paired with shock for avoidance animals as well as for Pavlovian animals, although CS + FS is paired with shock only for Pavlovian animals. Nor does the better performance of avoidance animals as compared with that of yoked controls require an instrumental interpretation; the yoked animals are activated to a lesser extent by the CS, which for them is paired with shock only inconsistently. It is of interest from this point of view to examine the performance of avoidance animals and their yoked controls under conditions in which shock is prevented by a specified amount of general activity rather than by a specified response.

\section{METHOD}

The Ss were 3-4-in. goldfish kept in individual 2-gal tanks, their water filtered, a erated, and te mperature-controlled. A detailed description of the apparatus is available elsewhere (Bitterman, 1966). The animals were trained in sets of six which could be treated as three yoked

* This work, supported by Grant MH-17736 from the U.S. Public Health Service, was done during the first author's tenure of a NATO postdoctoral fellowship. experiments was that, in the first, a response of the required magnitude not only prevented the US, but also terminated the $\mathrm{CS}$, while, in the second, the CS always stayed on throughout the CS-US interval. Yoked with each avoidance animal was a control animal which had exactly the same exposure to CS and US. After 13 days of acquisition, there were 7 days of extinction with avoidance and control animals unyoked. As before, response of the specified magnitude terminated the CS in the first experiment but not in the second.

\section{RESULTS}

The performance of 84 avoidance and control animals trained with CS termination in the first experiment is shown in Fig. 1; the performance of 60 animals trained without CS termination in the second experiment is shown in Fig. 2. The curves are plotted in terms of the mean probability of response (of the magnitude required for avoidance) on each day of acquisition and extinction. The two sets of data were subjected to analyses of variance, the outcomes of which are summarized in Table 1.

The first result of interest concerns the effectiveness of the US-avoidance contingency, which is demonstrated by the fact that the level of response was greater in the avoidance animals than in the yoked controls. Much of the difference in the acquisition curves of the first experiment may, of course, be attributed to unequal exposure to the CS, since the response of an avoidance animal terminated the CS both for itself and for the animal yoked with it, while the response of
ACQUISITION

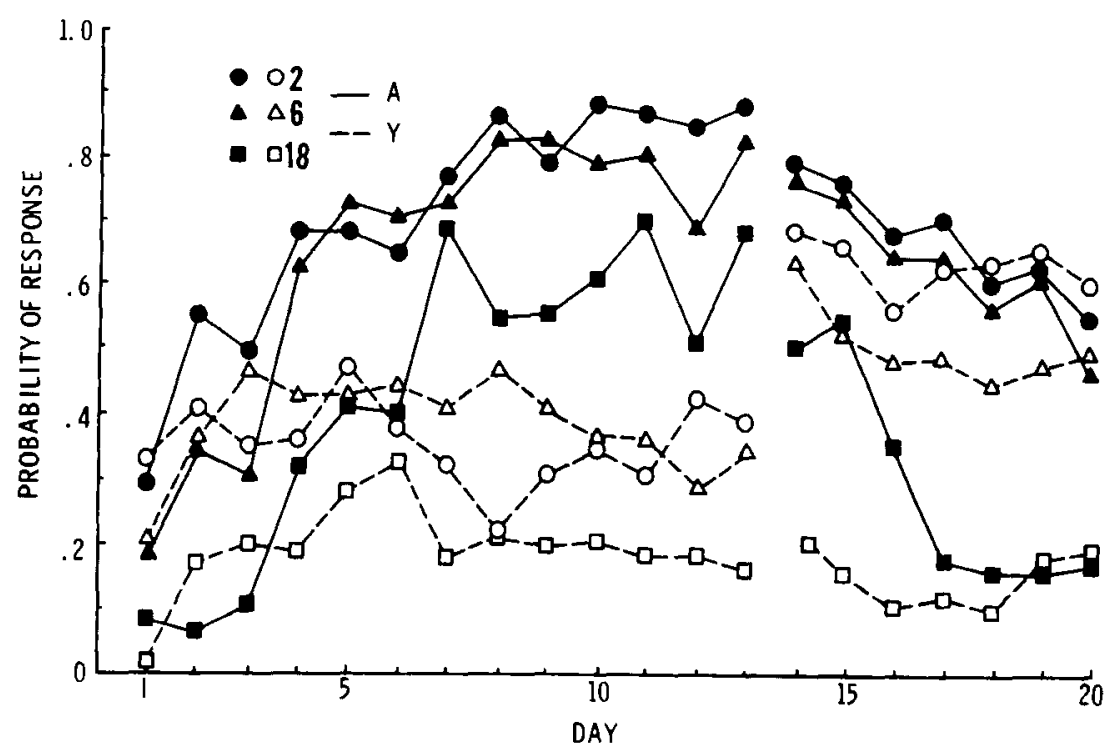

Fig. 1. The performance of the avoidance animals and their yoked controls trained with CS termination in the first experiment. 
Table 1

Analysis of Variance in Probability of a Response of Specified Magnitude by Avoidance Animals and Yoked Controls (Treatments) on Acquisition and Extinction Days in the Two Experiments

\begin{tabular}{|c|c|c|c|c|c|c|c|c|c|c|c|c|}
\hline \multirow[b]{3}{*}{ Source } & \multicolumn{6}{|c|}{ Experiment 1 (CS Termination) } & \multicolumn{6}{|c|}{ Experiment 2 (No CS Termination) } \\
\hline & \multicolumn{3}{|c|}{ Acquisition } & \multicolumn{3}{|c|}{ Extinction } & \multicolumn{3}{|c|}{ Acquisition } & \multicolumn{3}{|c|}{ Extinction } \\
\hline & df & $\mathbf{F}$ & $\mathbf{p}$ & df & $\mathbf{F}$ & $\mathbf{p}$ & df & $\mathbf{F}$ & $\mathbf{p}$ & df & $\mathbf{F}$ & $\mathbf{p}$ \\
\hline Treatments (T) & 1 & 68.97 & $<.01$ & 1 & 2.97 & 一 & 1 & 11.03 & $<.01$ & 1 & 3.75 & 一 \\
\hline Magnitudes (M) & 2 & 13.82 & $<.01$ & 2 & 29.17 & $<.01$ & 2 & 23.92 & $<.01$ & 2 & 99.34 & $<.01$ \\
\hline Days (D) & 12 & 20.74 & $<.01$ & 6 & 6.89 & $<.01$ & 12 & 24.20 & $<.01$ & 6 & 6.57 & $<.01$ \\
\hline $\mathrm{T}$ by $\mathrm{M}$ & 2 & $<1$ & - & 2 & $<1$ & - & 2 & 13.37 & $<.01$ & 2 & $<1$ & - \\
\hline $\mathbf{T}$ by $\mathbf{D}$ & 12 & 14.21 & $<.01$ & 6 & 2.72 & $<.05$ & 12 & $<1$ & - & 6 & 2.21 & $<.05$ \\
\hline M by $\mathbf{D}$ & 24 & $<1$ & - & 12 & $<1$ & - & 24 & 1.79 & $<.05$ & 12 & 1.70 & $<.05$ \\
\hline T by M by $D$ & 24 & 1.84 & $<.01$ & 12 & $<1$ & - & 24 & 1.26 & - & 12 & $<1$ & - \\
\hline
\end{tabular}

ACQUISITION

EXTINCTION

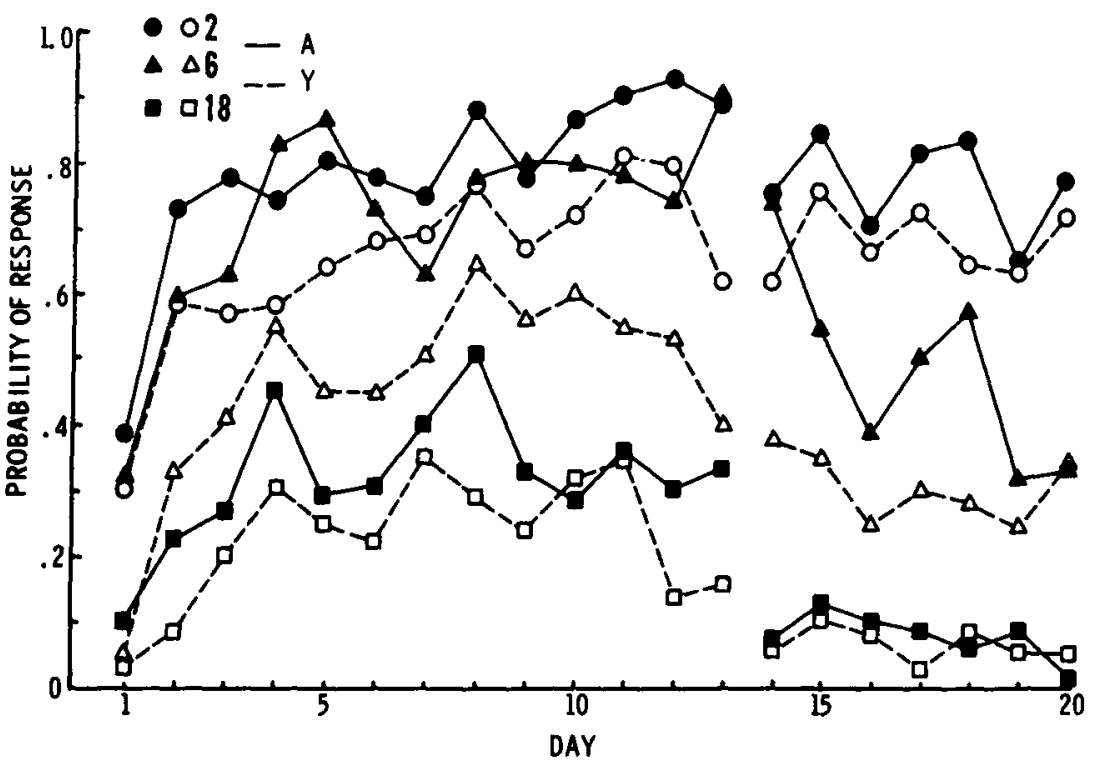

Fig. 2. The performance of the avoidance animals and their yoked controls trained without CS termination in the second experiment.

the yoked animal had no effect. In extinction, when the animals were unyoked and exposure to the CS thereby equated, the level of response in two of the control groups ( 2 and 6 units) increased precipitously. Nevertheless, a difference in resistance to extinction (as indicated by the significant Treatments by Days interaction) points to the effectiveness of the avoidance contingency. Further evidence is provided by the performance of the animals trained without CS termination, for which exposure to the CS was equal from the outset. The effectiveness of the avoidance contingency for these animals is indicated both by the significant treatments variance in acquisition and by the significant Treatments by Days interaction in extinction.

A second result of interest concerns the effectiveness of the CS termination contingency. While formal comparisons across the two experiments are difficult to make, the curves suggest that the performance of the avoidance animals was improved by CS termination, especially when the required magnitude of response was high. The results of variation in magnitude also are worth noting. Beyond the main effect, whose explanation is obvious, there is a significant interaction with treatments in the second experiment which indicates that the variable plays an important role in acquisition, at least when there is no CS termination contingency.

\section{DISCUSSION}

It seems clear that the general activity of goldfish can be conditioned by avoidance as well as by Pavlovian procedure. The results are compatible with the view that the increased probability of hurdle crossing produced by avoidance training in the shuttlebox is due to increased activation of the animal by the CS.
Both sets of results can be understood without reference to a process of instrumental learning on the assumption that the sensory feedback from the response which meets the avoidance requirement is readily discriminable. The fact that CS termination seems to play a more important role in the general activity situation than in the shuttlebox is attributable from this point of view to the better discriminability of the residual feedback from shuttling. An advantage of specifying the avoidance response may be that the difference between feedback from the behavior which avoids shock and the behavior which does not is enhanced, although it is conceivable that specifying only the amount of activity required for avoidance does in fact place some restriction on the kind of behavior which will meet the requirement, with the result that some amounts give better performance than others. There are, of course, several different reasons for expecting that performance will vary with the amount of activity required. Since the structure of the shuttlebox determines the relation between level of activation and probability of hurdle crossing, the results obtained in the particular shuttlebox thus far employed must depend to a considerable extent upon its dimensions. However fortuitous the choice of those dimensions may have been, the apparatus provides a convenient measure of conditioned activation in the goldfish.

\section{REFERENCES}

BITTERMAN, M. E. Animal learning. In J. B. Sidowski (Ed.), Experimental methods and instrumentation in psychology. New York: McGraw-Hill, 1966.

HORNER, J. L. LONGO, N., \& BITTERMAN, 'M. E. A classical conditioning technique for small aquatic animals. American Journal of Psy chology, $1960,73,623-626$.

WOODARD, W. T., BITTERMAN, M. E. Classical conditioning of goldfish in the shuttlebox. Behavior Research Methods \& Instrumentation, 1971, 3, 193-194.

WOODARD, W. T., \& BITTERMAN, M. E. A $\mathrm{Pavlovian}$ analysis of avoidance conditioning in the goldfish. Journal of Comparative \& Physiological Psychology, in press. 